MATEC Web of Conferences 25, 01011

(2015)

DOI: $10.1051 /$ matecconf/ 20152501011

(c) Owned by the authors, published by EDP Sciences, 2015

\title{
Numerical Analysis of Effect of Boundary Layer Characteristics on the Flow Field in S-shaped Inlet
}

\author{
Jia Ren, Naiming Wu, Mingzhen Li, Qiyang Hu \& He Liu \\ School of Energy and Power Engineering, Beihang University, Beijing, China
}

\begin{abstract}
In order to explore the effect of boundary layer thickness and pressure gradient on the performance of the flow field in the inlet, we design a high offset rate S-shaped inlet based on a certain unmanned aerial vehicle (UAV), and its author has analyzed the effect of boundary layer characteristics on the inlet with numerical simulation method. The suction of boundary layer which leads to separation zone not only becomes longer in the inlet, but also moves to the center plane of symmetry, the separation point of boundary layer appears in advance as pressure gradient increases. Considering the influence of the boundary layer, various performance parameters all exceeds that of the uniform entrance inlet conditions, especially the circumferential total pressure distortion of outlet increased by $58.2 \%$ at most, obviously can't meet the engine to work properly, so we must consider and pay attention to the effect of the boundary layer characteristics on the flow field in the S-shaped inlet.
\end{abstract}

Keywords: S-shaped inlet; boundary layer; separation; numerical simulation

\section{INTRODUCTION}

The engine inlet is an important component of the propulsion system; its performance quality directly affects the performance of the engine. Considering its aerodynamic layout constraints and invisibility performance requirements, S-shaped inlet has been widely concerned because of its good invisibility, compact structure, advantages of small windward area in recent years. However, flowing air flow in the $\mathrm{S}$-shaped pipe has adverse pressure gradient due to the particularity of its shape, and the inlet air flow is very difficult to stick attached to the bending of the pipe wall, so prone to ${ }^{[1]}$ separation. The flow separation phenomenon is resulted by the effect of centrifugal force, static pressure distribution form the " $\mathrm{X}$ " along the up and down the wall. There is serious secondary flow on circumferential because of the driving of the horizontal adverse pressure gradient, which can cause large outlet pressure distortion ${ }^{[3]}$. In addition, airframe boundary layer can also cause the inlet total pressure recovery coefficient decreased when the plane flights, it will cause the total pressure distortion and instability of airflow. Also, flow conditions will make change when the planes takeoff, land or change the flight condition, which can lead to the corresponding change inside of the inlet flow, further affect the performance of the engine parts and even the whole machine. The study of flow characteristic of inlet started early abroad; however, the in-depth study of S-shaped inlet begins with a jet plane, and then has been applied on the cruise missile ${ }^{[4-12]}$. Plas ${ }^{[13]}$ systematically studied the boundary layer suction for promoting the performance of the system, the results show that the boundary layer suction can reduce fuel consumption by a few percentage points, and the distortion along the piping is the main reason for the overall performance. Liu Zhenxia ${ }^{[14]}$ analyzed the influence of former fuselage of S inlet on the entrance and the shock wave distribution of internal flow field based on the simulation of supersonic flow field. Wang Xiao ${ }^{[15]}$ studied the three dimensional viscous flow field numerical simulation based on five specific short $\mathrm{S}$ inlets using CFX software, the results show that even increase the length of the straight segment, such S-inlet is also difficult to delay flow separation. However, the current research on characteristic of $\mathrm{S}$ flow field in the inlet boundary layer is not enough.

The purpose of this paper is to study the influence of inlet boundary layer on the characteristic of S flow field using the method of numerical simulation. Furthermore, this paper is also used to analyze the influence of boundary layer thickness in the variable operating conditions and the pressure gradient curve in the inlet boundary layer on the characteristic of $\mathrm{S}$ flow field, including the total pressure recovery coefficient, the total pressure distortion of outlet and so on.

\section{PHYSICAL MODEL}

\subsection{Geometric model}

Based on a certain S-shaped inlet on the back of unmanned aerial vehicle, in order to increase the tank volume and reduce the plane empty weight ${ }^{[16]}$, we design a runway-shaped entrance for inlet; the outlet is a circle with a diameter of $100 \mathrm{~mm}$, and the total length of S-shaped inlet is $377 \mathrm{~mm}$. In addition, the expansion ratio of the import and export is 1.4 , and the

This is an Open Access article distributed under the terms of the Creative Commons Attribution License 4.0, which permits unrestricted use, distribution, and reproduction in any medium, provided the original work is properly cited. 


\section{MATEC Web of Conferences}

S-shaped inlet offset rate is 0.35 , as shown in Figure 1.

Considering the inlet on the vertical center section of the fuselage is symmetrical, this paper does not involve the sliding condition, so we choose half of the cross section as the computational domain area. Due to subsonic flow in the pipe, outlet's boundary condition will affect the flow of the upstream, so the computational domain is supposed to be equivalently extended to about one fold the outlet, insuring that it is consistent with the actual flow as far as possible.

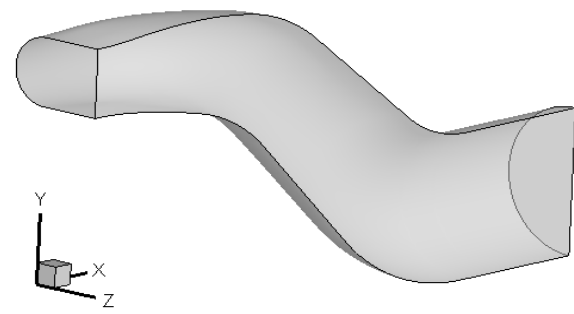

Figure 1. Geometry model of S-shaped inlet

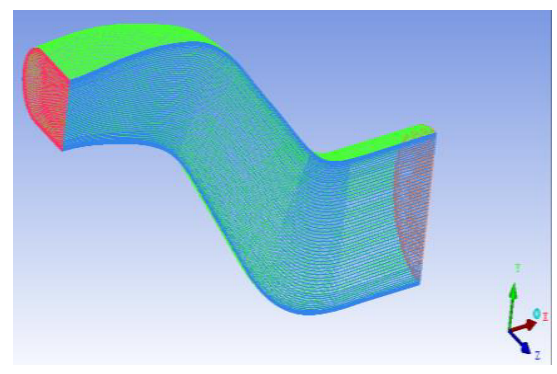

Figure 2. 137w structured grids

\subsection{Computational grids}

To split the grids based on the established model using ICEM software with structured grids, creating seven sets of grids in different numbers respectively, which include $18 \mathrm{w}, 27 \mathrm{w}, 40 \mathrm{w}, 61 \mathrm{w}, 91 \mathrm{w}, 137 \mathrm{w}$ and 206 w. The independent solution validation of grids is based on the condition of the entrance Mach number which is 0.5 . In this case, the inlet Mach number increases with the increase of grid number. But the results show that the grids number is over $137 \mathrm{w}$, Mach number no longer increases and the flow field changes with increasing the number of grid, therefore we study a numerical simulation using the grid number with $137 \mathrm{w}$. Also, reasonable boundary layer thickness is set up to ensure the value $y+$ of the first layer of grid which is 1 or so. In order to simulate the influence of the boundary layer shearing action and S-shaped inlet unique geometrical configurations on secondary flow more accurately, we adopt grid refinement processing along the wall of S-shaped including inlet and outlet, and the grid model is shown in Figure 2.

\subsection{Boundary conditions}

Considering the impact of boundary layer suction, set up total pressure of inlet under different conditions with User-defined-function (UDF), because the numerical simulation model of this paper is based on subsonic wind tunnel experiment of the Beijing University of Aeronautics and Astronautics Thermal Power Research Institute, and the inlet and outlet boundary conditions are set by the given experimental parameters. The outlet's back pressure is given in accordance with local atmospheric pressure.

\subsection{Calculation method}

Numerical simulation is adapted based on N-S equation solver using the finite volume method, thus we choose implicit format in order to accelerate convergence to improve computational efficiency. Turbulence model is SST K-W model using second order upwind discretization, we set a rule that residuals of each equation decreased to five orders of magnitude as the criteria of convergence, while ensuring that the inlet and outlet flow has stabilized.

\section{ANALYSIS PROJECT}

Because the boundary layer in the circumferential direction is far less than the radial inhomogeneity, even negligible, this study does not consider the non-uniformity of boundary layer in the circumferential direction. In order to study the impact of different flow boundary layer thickness on the flow field inlet, we deduced total pressure boundary layer equations according to the speed boundary layer $(1)^{[17]}$ :

$-\frac{\Delta p^{*}}{L^{2}} y^{2}=X$

$\Delta \mathrm{p} *$ represents the difference between total pressure of flow and the total pressure of main wall, i.e. $\Delta$ $\mathrm{p} *=\mathrm{P} 0 *-\mathrm{P} 2 *$; L is the geometric thickness of the boundary layer; $y$ is the radial direction; $x$ is the spanwise direction.

Inlet's boundary layer parameters are set as follows in Table 1, project A00 is no boundary layer situation, then the total pressure distribution of inlet is constant in the radial distribution, as shown in Figure 3. Typical inlet's boundary layer pressure distribution in the radial direction is shown in Figure 3, and it is the quadratic curve distribution ${ }^{[19]}$. Figure 3 is the boundary layer parameters of project I . The project maintains boundary layer thickness in the radial height (i.e. the distribution curve ordinate value) the same by adjusting the value of quadratic curve in the horizontal axis (i.e. the minimum inlet total pressure is closer to the end wall) to change the boundary layer inlet total pressure curve distribution, and the project includes A11, A12 and A13. Figure 4 shows the inlet boundary layer parameters distribution of project II. The project 
is keeping the value of the horizontal axis of the distribution curve (i.e. the minimum total pressure of inlet is closer to the end wall) constant by varying flow boundary layer thickness in radial height to change the total pressure curve distribution of the boundary layer, and the project includes A21, A22 and A23, namely $\Delta \mathrm{h}$ decreases. A33 represents for $30 \%$ of the total height of the boundary layer thickness, and the pressure gradient is linear distribution.

\section{NUMERICAL ANALYSIS RESULTS}

\subsection{Static pressure coefficient along the wall}

Figure 5 shows the static pressure coefficient distribution curve along the up and down of the wall in inlet flow field. Both the $\mathrm{X}$-coordinate and $\mathrm{Y}$-coordinate have been dimensionless. $\mathrm{X}$-coordinate is $\mathrm{X} / \mathrm{D}$, and $\mathrm{D}$ is the diameter of the outlet; Y-coordinate is the static pressure coefficient along the wall, and the static pressure coefficient is defined as Equation (2):

$C p=\frac{p-p_{\infty}}{q_{\infty}}$

Table 1. Inlet boundary layer parameters

\begin{tabular}{llllll}
\hline Project I & $\Delta \mathrm{P}^{*} / \mathrm{pa}$ & $\mathrm{L} / \mathrm{h}$ & Project II & $\Delta \mathrm{P}^{*} / \mathrm{pa}$ & $\mathrm{L} / \mathrm{h}$ \\
\hline A11 & 11618 & $20 \%$ & A21 & 11618 & $10 \%$ \\
A12 & 5809 & $20 \%$ & A22 & 11618 & $20 \%$ \\
A13 & 1161 & $20 \%$ & A23 & 11618 & $30 \%$ \\
A33 & 11618 & $30 \%$ & Linear distribution & \\
A00 & \multicolumn{5}{|l}{ No boundary layer } \\
\hline
\end{tabular}

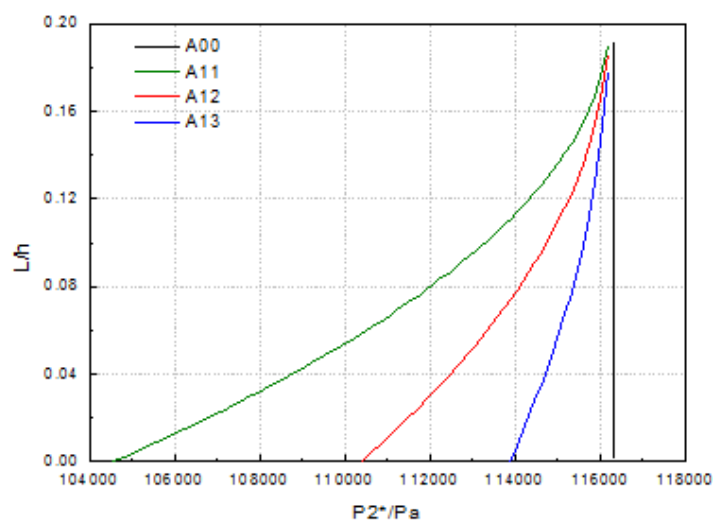

Figure 3. Project I of boundary layer

$\mathrm{P}$ is the static pressure along wall; $p_{\infty}$ is the static pressure of the incoming flow; $q_{\infty}$ is the dynamic pressure. Because S-shaped inlet bent down under the action of centrifugal force, more airflow concentrated near the upper wall and the pressure near the upper wall is higher than which is near the lower wall, and there is a large area of low pressure near the lower wall. It will produce a sharp increase of pressure before entering the second corner, and the upper wall is covered, so that airflow gets close to the lower wall where a significant low pressure area exists. In addition, there is a flat pressure gradient between two bends, that is, a so-called pressure platform. After the platform, the pressure of upper and lower wall gradually decreases until it is approximately equal to the same, and the entire distribution also forms an $\mathrm{X}$-shaped ${ }^{[18]}$. By comparison, we can found that the pressure plateau of the project A33 is ahead of $70 \mathrm{~mm}$ than project $\mathrm{A} 00$; the length of the flat area also continued to be longer, and the $\mathrm{Cp}$ of outlet is reduced from 0.6 to 0.4. Compare the project A11, A12 and A13, we can notice that the pressure platform gradually delayed occurred and the length of the pressure plateau is shortened, even the outlet's Cp is improved from A11 to A33. Furthermore, the length of the pressure plateau is longer as the pressure gradient is higher. Compared with project II, Cp keeps the same along the first curve, but the position of separation takes place at the same location along the flow direction after entering the second corner; $\mathrm{Cp}$ of outlet is decreased from A21 to A23, because the low-pressure area increases as thickness of the boundary layer increases; $\mathrm{Cp}$ of the project A23 is less than other projects.

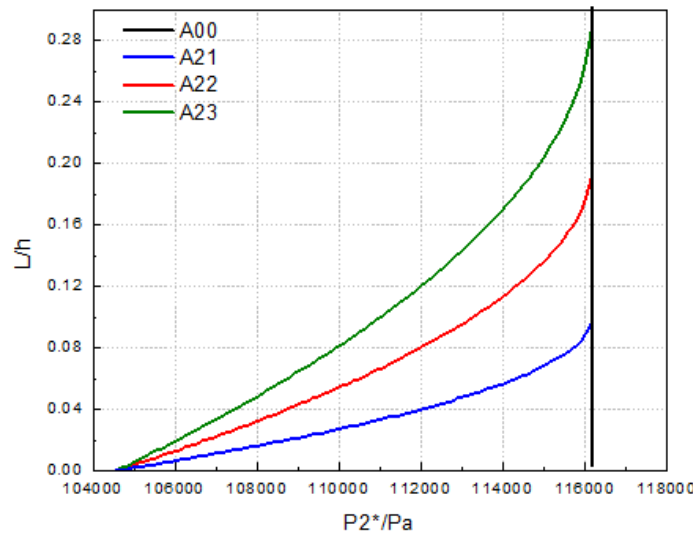

Figure 4. Project II of boundary layer

4.2 Separation zone along spanwise direction and flow direction

Numerical simulation is based on uniform inlet boundary conditions and non-uniform inlet boundary condition under $\mathrm{Ma}=0.66$ in order to describe the boundary layer at the lower wall more directly. Figure 6 shows the nephogram and streamline of $\Delta \mathrm{Z} / \mathrm{D}=0$, $0.16,0.25$ and 0.3 in lower wall, in which the $\Delta \mathrm{Z}$ is the distance from the center of symmetry plane to the 


\section{MATEC Web of Conferences}

spanwise direction. The simulation results of uniform inlet boundary condition show that separation occurs after the first bend, the largest separation zone appears in $\Delta \mathrm{Z}=0.25 \mathrm{D}$ plane, and the separation point locates in $\mathrm{x}=-0.15$ along the flow direction. It illustrates that the internal flow field separation does not appear in the center plane of symmetry, but $25 \mathrm{~mm}$ in spanwise direction from the symmetry plane. Table 2 shows the separation zone distribution along flow direction and spanwise direction.

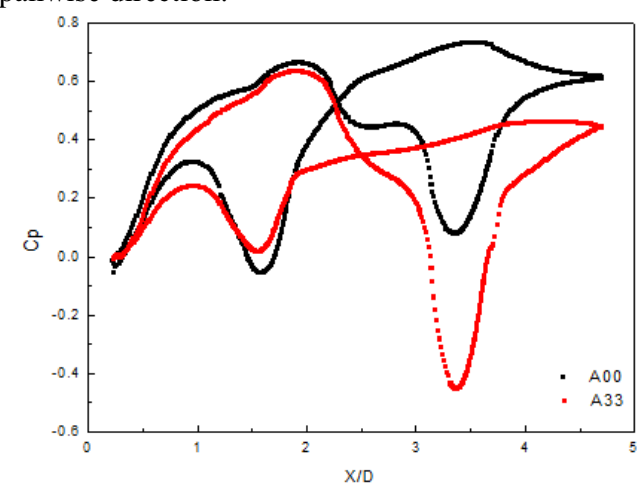

(a)

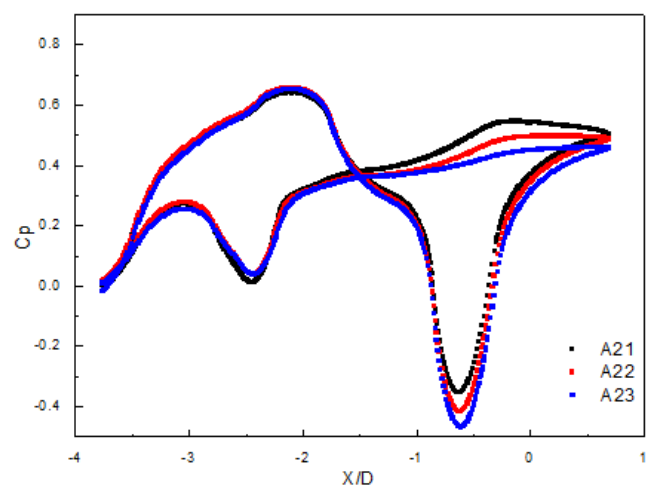

(b)

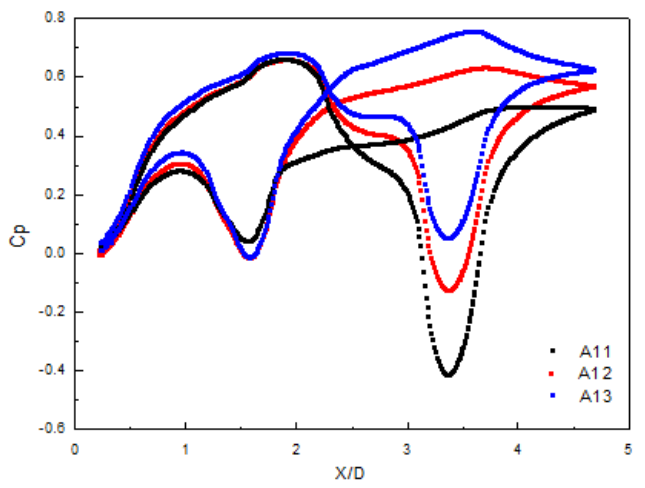

(c)

Figure 5. Static pressure coefficient along upper and lower wall
There are three different pressure gradient distributions in project I. The streamline shows that the largest separation zone locates at $\Delta \mathrm{Z}=0.16 \mathrm{D}$, indicating that boundary layer fluid rolled the low-energy fluid toward the center, and its separation location is closer to the center. But three conditions of project I are on the same position along spanwise direction, indicating that different pressure distribution in the boundary layer does not affect separation along spanwise direction. The boundary layer height of project II can cause separation zone changed along the spanwise direction. Finally, the separation zone of project A33 in the linear distribution and A21 are the closest to the center position along spanwise direction, which located in $\Delta$ $\mathrm{Z}=0.10 \mathrm{D}$

Boundary layer can cause separation of the air flow in advance; the flow field separation point of uniform inlet boundary condition locates in $X=-0.15 \mathrm{~m}$; the flow field separation points of non-uniform inlet boundary condition are also ahead. Project I showed that the separation point appears ahead as the pressure gradient of boundary layer becomes higher. Project II shows that the area of separation is not larger with the increasing height of the boundary layer increased. In conclusion, compared with other project, project A33 in linear distribution caused a great impact on separation along spanwise direction and flow direction.

\subsection{Total pressure recovery coefficient nephogram}

The boundary layer separation in S-shaped inlet will consume a lot of energy, which can cause low pressure to be closer to the lower wall, reducing the inlet total pressure recovery coefficient and resulting in greater outlet total pressure distortion. Total pressure recovery coefficient is defined as Equation (3):

$$
\sigma=\frac{p_{\text {out }}^{*}}{p_{\text {in }}^{*}}
$$

$p_{\text {out }}^{*}$ is the average total pressure of outlet and $p_{\text {in }}^{*}$ is the average total pressure of inlet. Compared with various projects, the total pressure recovery nephogram of outlet illustrates that the low-pressure area is near the upper wall and the lower wall surface, but the low pressure area which is near lower wall is much larger than the area which is near upper wall because of under the influence of flow separation. Project I and the total pressure recovery coefficient nephogram of outlet were not significantly different, showing that the distribution of the pressure gradient of the outlet has little effect on total pressure recovery coefficient. Only the values of different pressure lead to different pressure recovery coefficient of outlet. Compared with project II as shown in Table 2, the whole pressure recovery of outlet is higher and a low pressure area which forms an oval shape is closer to the lower wall of outlet, because the boundary layer thickness is thin; project A22 shows that the low value of total pressure 

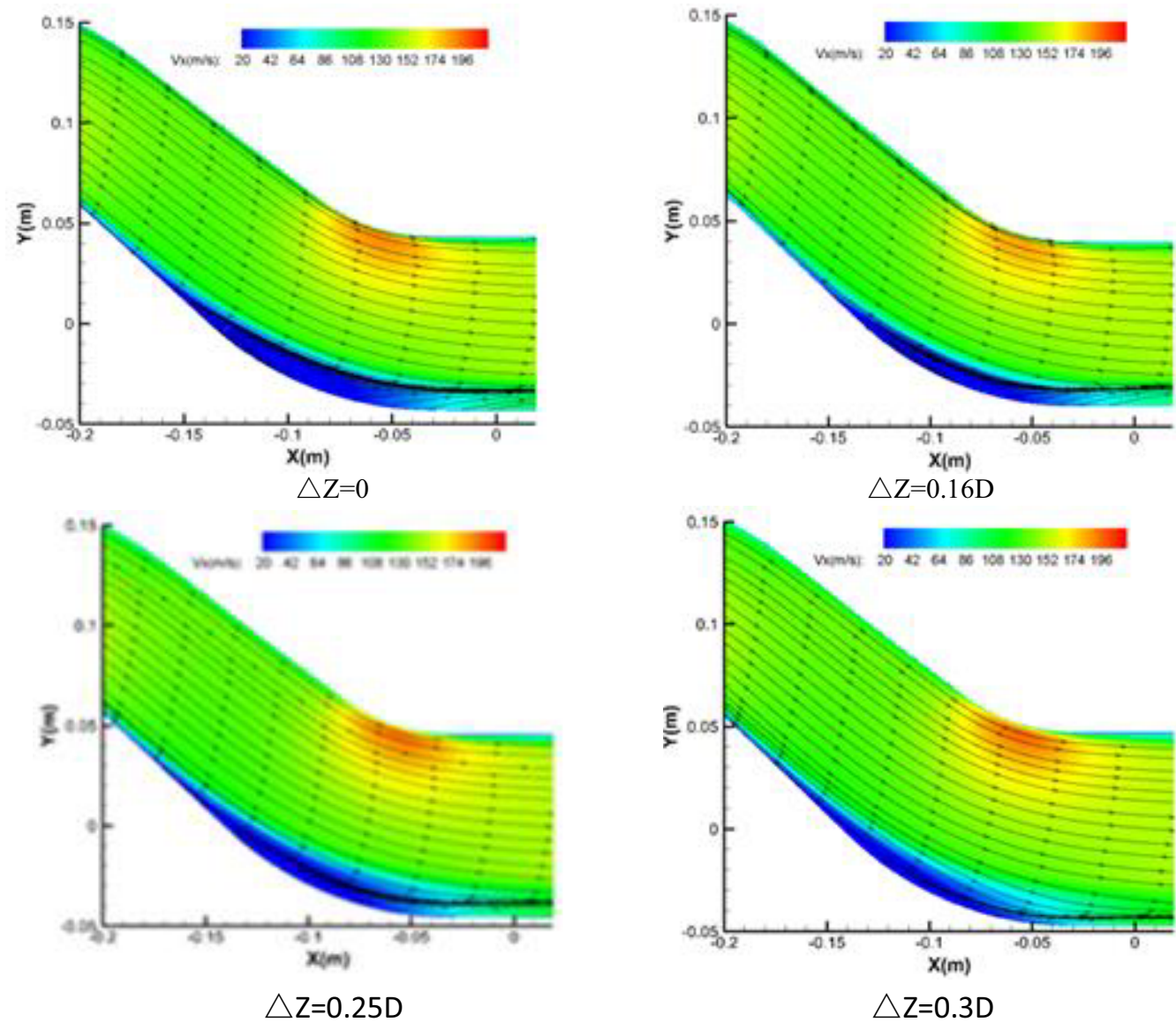

Figure 6. Velocity nephogram and streamline of different plane along spanwise direction

Table 2. Separation along spanwise direction and flow direction of different project

\begin{tabular}{llllll}
\hline Project I & $\begin{array}{l}\text { Spanwise location } \\
\Delta \text { Z/D }\end{array}$ & $\begin{array}{l}\text { Flow location } \\
\mathrm{X}(\mathrm{m})\end{array}$ & Project II & $\begin{array}{l}\text { Spanwise location } \\
\Delta \mathrm{Z} / \mathrm{D}\end{array}$ & $\begin{array}{l}\text { Flow location } \\
\mathrm{X}(\mathrm{m})\end{array}$ \\
\hline A11 & 0.16 & -0.22 & $\mathrm{~A} 21$ & 0.10 & -0.20 \\
$\mathrm{~A} 12$ & 0.16 & -0.16 & $\mathrm{~A} 22$ & 0.16 & -0.22 \\
$\mathrm{~A} 13$ & 0.16 & -0.14 & $\mathrm{~A} 23$ & 0.16 & -0.20 \\
$\mathrm{~A} 33$ & 0.10 & -0.22 & A00 & 0.25 & -0.15 \\
\hline
\end{tabular}

recovery coefficient is near the wall, but the low pressure area is not concentrated. The total pressure recovery coefficient of project A23 is similar to the project A21 in shape, but the height of low pressure is clearly higher than the above, accounting for $50 \%$ of the overall height. Also, the area is the largest. In summary, with increasing boundary layer thickness, the height of the low-pressure zone and the area increases.

\subsection{Total pressure distortion of outlet and pressure irregularity}

Circumferential pressure distortion index DC60 is the difference of average total pressure and the minimum average total pressure within the cross-section area divided by the dynamic pressure of outlet, it is a parameters evaluating irregularity and generally uses $60^{\circ}$ fan-shaped region as a reference ${ }^{[19]}$, the expression is an Equation(4):

$D C_{60}=\frac{P_{\text {out }}^{*}-P_{\text {out }, \min (60)}^{*}}{q_{\text {out }}}$

Irregularity of outlet is as follows:

$$
D=\frac{P_{\text {out }, \max }^{*}-P_{\text {out }, \text { min }}^{*}}{P_{\text {out }}^{*}}
$$



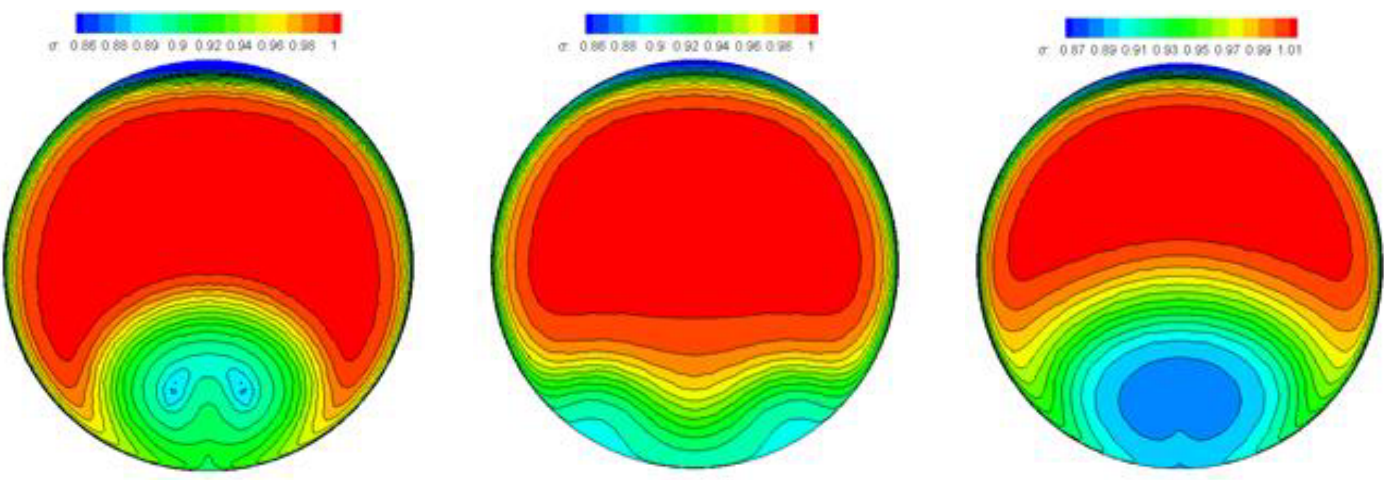

Figure 7. Total pressure recovery coefficient nephogram of outlet

$$
P_{\text {out } \text { max }}^{*} \text { is the maximum of total pressure; } P_{\text {out, } \min }^{*} \text { is }
$$

the minimum of total pressure; $P_{\text {out }}^{*}$ is the average total pressure of outlet.

$$
\sigma=\frac{p_{\text {out }}^{*}}{p_{\text {in }}^{*}} \text { is the total pressure recovery coeffi- }
$$

cient, performance parameters of inlet in different projects are shown in Table 3.

Table 3 shows that the boundary layer will cause the total pressure recovery coefficient of outlet section to be reduced and the circumferential total pressure distortion becomes large. Because of the suction of boundary layer, total pressure of the flow field in inlet is lower than the uniform inlet flow field, and the maximum total pressure value on the outlet section is not high, resulting in the value of irregularity of project I and II which is less than that of uniformity. Boundary layer has a significantly affected on circumferential total pressure distortion index, resulting in the value of which exceeds the standard value. Therefore, we should take effective control measures to improve the quality of inlet.

Table 3. Performance parameters of inlet in different projects

\begin{tabular}{llll}
\hline Project & $\sigma$ & $\mathrm{D}$ & $\mathrm{DC}_{60}$ \\
\hline $\mathrm{A} 00$ & 0.9871 & $18.7 \%$ & $6.9 \%$ \\
$\mathrm{~A} 11$ & 0.9868 & $16.4 \%$ & $47.9 \%$ \\
$\mathrm{~A} 12$ & 0.9866 & $16.3 \%$ & $29.9 \%$ \\
$\mathrm{~A} 13$ & 0.9862 & $14.8 \%$ & $6.7 \%$ \\
$\mathrm{~A} 21$ & 0.9829 & $16.3 \%$ & $32.4 \%$ \\
$\mathrm{~A} 22$ & 0.9868 & $16.4 \%$ & $47.9 \%$ \\
$\mathrm{~A} 23$ & 0.9888 & $16.5 \%$ & $65.1 \%$ \\
$\mathrm{~A} 33$ & 0.9874 & $16.1 \%$ & $45.5 \%$ \\
\hline
\end{tabular}

\section{CONCLUSION}

This paper discusses the influence of the boundary layer on the flow field and performance of the $\mathrm{S}$ inlet. The conclusions are as follows:

(1) Suction of the boundary layer results in platform of static pressure coefficient curve along the wall appears early, and the length of separation zone becomes longer. In addition, the separation point appears in advance with increasing gradient of boundary layer pressure. But the size of separation zone is not changed with thickness of boundary layer.

(2) The boundary layer affects a lot on separation zone distribution along the spanwise direction; the low energy fluid concentrated near the position is $10 \mathrm{~mm}$ to $16 \mathrm{~mm}$ off the symmetrical faces because of suction of boundary layer. However, the position will not be changed as an increase with the gradient boundary layer pressure.

(3) The boundary layer thickness can cause the total pressure recovery coefficient of outlet to change, the low pressure area of the outlet section and the height will increase with the thickness of the boundary layer increases. When the boundary layer thickness is $30 \%$, the overall height of the low pressure area is $50 \%$. But the pressure gradient changes did not cause the total pressure recovery coefficient to change significantly.

(4) The total pressure recovery coefficient is reduced by $0.4 \%$ at maximum under the influence of inlet boundary layer, especially the circumferential total pressure distortion which is increased by $58.2 \%$ at maximum, and it has been unable to meet the engine requirement. Therefore, the study about measures to control the influence of layer on the flow field is significant.

\section{REFERENCES}

[1] Chen Zhan-jun, 2012. The study of synthetic jet improving the S-shaped inlet flow characteristics. China Science: Technology Science, 42(9):1054-1060.

[2] J Seddon, \& E L Goldsmith. 1999. Intake Aerodynamics. London: Blackwell Science. 
[3] Zhu Yu, Luo Chang-tian, \& Wang Xiao, 2005. Ultra-compact inlet airflow control technology. Aeroplane Design. (4):1-6.1

[4] L.G. Hunter, \& J. E. Hawkins. 1981. F-16 Variable-Geometry Inlet Design and Performance. AIAA 81-1394.

[5] L. E. Surber, \& Lt. C. P. 1983. Robinson. Survey of Inlet Development for Supersonic Tactical Aircraft. AIAA 83-1164,

[6] V.Lyman, \& J. P. Hancock. 1986. Correlation of Theory and Experiment for Propfan Inlets. AIAA 1986-1628,

[7] J. J. Korte, D. J. Singh, A. Kumar, \& A. H. Auslender. 1994. Numerical Study of the Performance of Swept, Curved Compression Surface Scramjet Inlets. Journal of Propulsion and Power. 10(6)

[8] John Fleming, \& Jason Anderson. 2005. Sensing and Active Flow Control for Advanced BWB Propulsion-Airframe Integration Concepts, NASA/CR -213919.

[9] J. M. Abbott, \& J. W. Slater. 2001. Computational Study of the Aerodynamic Performance of Three-Dimensional Subsonic Inlets. AIAA 2001-3886.

[10]B. L. Berrier, \& B. G. Allan. 2004. Experimental and Computational Evaluation of Flush Mounted, S-Duct Inlets[R]. AIAA 2004-764.

[11]A. M. Kirk, A. Kumar, \& J. I. Gargoloff. 2007. Numerical and Experimental Investigation of a Serpentine Inlet Duct. AIAA 2007-842.
[12]O. Basoglu, L. Yalcin, \& A. E. Yilmaz. 2008. Computational Study on the Design Parameters of S-Type Subsonic Inlets. AIAA 2008-73.

[13]A. P. Plas, M. A. Sargeant, V. Madani, D. Crichton, E. M. Greitzer, T. P. Hynes, \& C.A. Hall. 2000. Performance of a Boundary Layer Ingesting Propulsion System. AIAA 2007-450,

[14]Liu Zhen-xia, Guo Dong-ming, Zhang Li-fen, \& Wu Ding-yi. 2006. Numerical Simulation of S-shaped inlet .Paper of Aerospace Dynamic. (06)

[15]Zhu Yu, \& Wang Xiao. 2004. Numerical Simulation of short S-shaped inlet. Aerospace Design. (04)

[16] Hamstra J W, Miller D N, \& Truax P P, et al. 2000. Active inlet flow control technology demonstration. Aeronaut, 104(1040): 473-479

[17]Zhang Yong-jun, \& Feng Guo-tai. The influence of imported boundary of planar curved blade diffuser on the performance of Cascade Flow. Paper of Aerospace Dynamic, 006, 21(2): 254-260.

[18]He Peng, \& Dong jin-zhong, 2015. Effects of synthetic jet layout on S-shaped inlet separation control, Paper of Aerospace Dynamic, Vol. 30 No.2 Feb.

[19]Chen Jian-hua, 2009. Study of boundary layer suction inlet with active flow control, Harbin Industry University. 